\title{
Controlling transition probability from matter-wave soliton to chaos
}

\author{
Qianquan Zhu, Wenhua Hai*, Shiguang Rong \\ Key Laboratory of Low-dimensional Quantum Structures and Quantum Control of Ministry of Education, and \\ Department of physics, Hunan Normal University, Changsha 410081, Chind
}

\begin{abstract}
For a Bose-Einstein condensate loaded into a weak traveling optical superlattice it is demonstrated that under a stochastic initial set and in a given parameter region the solitonic chaos appears with a certain probability. Effects of the lattice depths and wave vectors on the chaos probability are investigated analytically and numerically, and different chaotic regions associated with different chaos probabilities are found. The results suggest a feasible method for eliminating or strengthening chaos by modulating the moving superlattice experimentally.
\end{abstract}

PACS numbers: 03.75.Lm, 05.45.Ac, 03.75.Kk, 05.45.Gg

Many phenomena observed in Bose-Einstein condensates (BECs) are well modelled by nonlinear Schrödinger Equation (NLSE), also known as Gross-Pitaevskii equation (GPE), which includes many fantastic nonlinear effects, such as chaos [1, 2], soliton [3, 4], and so on. Chaotic soliton behaviors, which are of particular interest, have been studied theoretically in a NLSE with a periodic perturbation [5, 6, 7]. Lately, there are a few remarkable works on chaotic dynamics of soliton in BEC systems, including the chaos and energy exchange [8], the discrete soliton and chaotic dynamics in an array of BECs [9], and the bright matter-wave soliton collision [10]. Recently, increasing interest is excited in a BEC held in an optical superlattices for the periodic 11, 12] and quasiperiodic 13, 14, 15] cases which are in close analogies with the fields of supercrystals and quasicrystals [16]. The BECs interacting with a traveling lattice have also been successfully treated experimentally [17, 18] and theoretically [19, 20, 21] that shows many fantastic results such as lensing effect 18], gap soliton generation [19], spatiotemporal chaos 20, 21], and so on. From the above analyzes we get a physical motivation, namely using a moving optical superlattices to study the transitions from soliton to chaos in BEC matter.

It is well known that for stochastic initial and boundary conditions and fixed parameters, chaos does not always appear in a chaotic system[20], but with certain probability. Chaos probability may play a significant role for the control of chaos. Many works have focused on suppressing chaos which results in zero chaos probability. Whereas, in some realistic applications such as secure communication based on chaos 22], higher chaoticity is desired [23], which calls for higher chaos probability. In this paper, we show that in a BEC system perturbed by a moving optical superlattice consists of two lattices of different depths and wave vectors, the superlattice can separate the chaotic region into several parts with different chaos probabilities. Furthermore, for a fixed first lattice the adjustment to the secondary lattice could turn the

${ }^{*}$ Corresponding Author: whhai2005@yahoo.com.cn chaos probability to zero or higher one. The results suggest a feasible method for eliminating or strengthening chaos experimentally. Such a method can be extended to the controls of the spatial chaos with zero traveling velocity and the temporal chaos in other systems.

Primary and secondary moving optical lattice compose the superlattice 24] as the form

$$
V_{o p}(\zeta)=V_{1} \cos ^{2}(k \zeta)+V_{2} \cos ^{2}(\gamma k \zeta+\phi)
$$

where we refer to $V_{1} \cos ^{2}(k \zeta)$ as the primary lattice with $V_{1}$ and $k$ corresponding to its depth and wave vector, and $V_{2} \cos ^{2}(\gamma k \zeta+\phi)$ as the secondary one with $V_{2}$ being its depth, $\gamma$ the ratio of the two laser wave vectors and $\phi$ the phase difference. The spatiotemporal variable $\zeta=x+v_{L} t$ contains the velocity of the traveling lattice $v_{L}=\delta /(2 k)$ with $\delta$ being the frequency difference between the two counter-propagating laser beams producing the first lattice. The laser frequencies and amplitudes can be controlled independently by using the acousto-optic modulators [17].

Chaotic Solitons For the quasi-one-dimensional (1D) BEC held in superlattice potential $V_{o p}(\zeta)$ the transverse wave function is approximately in ground state of a harmonic oscillator of frequency $\omega_{r}$, and the governing timedependent GPE reads

$$
i \frac{\partial \Psi}{\partial t}=-\frac{\hbar^{2}}{2 m} \frac{\partial^{2} \Psi}{\partial x^{2}}+g_{1 d}|\Psi|^{2} \Psi+V_{o p}(\zeta) \Psi .
$$

Here $\Psi$ and $m$ are the macroscopic quantum wave function and mass, $g_{1 d}=2 \hbar \omega_{r} a_{s}$ characterizes the 1D interatomic interaction strength [25] with $a_{s}$ being the s-wave scattering length. In order to get a simple description, we investigate the traveling wave solution in the form [20]

$$
\Psi=R(\zeta) e^{i[\theta(\zeta)+\alpha x+\beta t]},
$$

where $R(\zeta)$ and $\theta(\zeta)$ are real functions of $\zeta, \alpha$ and $\beta$ represent two undetermined real constants. Inserting Eq.(3) into Eq.(2), we easily obtain two coupled equations

$$
\begin{aligned}
\frac{d^{2} R}{d \xi^{2}} & -R\left(\frac{d \theta}{d \xi}\right)^{2}-(v+2 \tilde{\alpha}) R \frac{d \theta}{d \xi}-\left(\tilde{\beta}+\tilde{\alpha}^{2}\right) R-g_{1} R^{3} \\
& =\left[\tilde{V}_{1} \cos ^{2}(k \xi)+\tilde{V}_{2} \cos ^{2}(\gamma k \xi+\phi)\right] R, \\
R \frac{d^{2} \theta}{d \xi^{2}} & +2 \frac{d R}{d \xi} \frac{d \theta}{d \xi}+(v+2 \tilde{\alpha}) \frac{d R}{d \xi}=0 .
\end{aligned}
$$


In the formulas we have used the dimensionless variables and parameters $\xi=k_{0} \zeta, v=2 m v_{L} / \hbar k_{0}, \tilde{\beta}=$ $\hbar \beta / E_{r 0}, \tilde{\alpha}=\alpha / k_{0}, \quad \tilde{V}_{1}=V_{1} / E_{r 0}, \quad \tilde{V}_{2}=V_{2} / E_{r 0}, E_{r 0}=$ $\hbar^{2} k_{0}^{2} /(2 m)$ and $g_{1}=4 a_{s} /\left(k_{0} l_{r}^{2}\right)$ with $k_{0}$ being the unit of wave vector and $l_{r}=\sqrt{\hbar /\left(m \omega_{r}\right)}$ the radical length of harmonic oscillator. Integrating Eq.(5) yields $d \theta / d \xi=$ $C / R^{2}-(v / 2+\tilde{\alpha})$, where $C$ is the integration constant. Applying this to Eq.(5), we arrive at the decoupled equation

$$
\begin{aligned}
& \frac{d^{2} R}{d \xi^{2}}-\frac{C^{2}}{R^{3}}+D R-g_{1} R^{3} \\
& =\left[\tilde{V}_{1} \cos ^{2}(k \xi)+\tilde{V}_{2} \cos ^{2}(\gamma k \xi+\phi)\right] R
\end{aligned}
$$

with $D=\frac{1}{4} v^{2}+v \tilde{\alpha}-\tilde{\beta}$. It is very hard to find the exact solution of this equation. However, when the driving strengths are weak enough, we can treat the chaotic system by the direct perturbation approach 26 and Melnikov-function method [27].

For simplicity we consider the $C=0$ case which leads Eq. (6) to the driven Duffing equation, whose chaotic features have been extensively studied for the single lattice case 20, 21]. In the double lattice case, we expand $R$ to the first order,

$$
R(\zeta)=R_{0}(\zeta)+R_{1}(\zeta), \quad\left|R_{0}\right| \gg\left|R_{1}\right|,
$$

and insert it into Eq.(6) with $C=0$, obtaining the zeroth-order and first-order equations

$$
\begin{gathered}
\frac{d^{2} R_{0}}{d \zeta^{2}}+D R_{0}-g_{1} R_{0}^{3}=0, \\
\frac{d^{2} R_{1}}{d \xi^{2}}+D R_{1}-3 g_{1} R_{0}^{2} R_{1}=\varepsilon(\xi), \\
\varepsilon(\xi)=\left[\tilde{V}_{1} \cos ^{2}(k \xi)+\tilde{V}_{2} \cos ^{2}(\gamma k \xi+\phi)\right] R_{0} .
\end{gathered}
$$

If the atom-atom interactions are attractive, the system has a negative $s$-wave scattering length to make $g_{1}<0$ such that Eq.(8) with a negative $D$ has the well known homoclinic solution

$$
\begin{aligned}
& R_{0}(\xi)=\sqrt{\frac{2 D}{g_{1}}} \operatorname{sech}\left[\sqrt{-D}\left(\xi+c_{0}\right)\right], \\
& c_{0}=\frac{1}{\sqrt{-D}} \operatorname{Ar} \operatorname{sech}\left[\sqrt{\frac{g_{1}}{2 D}} R_{0}\left(\xi_{0}\right)\right]-\xi_{0} .
\end{aligned}
$$

Here $\xi_{0}=k_{0}\left(x_{0}+v_{L} t_{0}\right)$ is the combination of the initial time $t_{0}$ and boundary coordinate $x_{0}, c_{0}$ denotes an integration constant determined by the boundary and initial conditions. Obviously, the zeroth-order number density $R_{0}^{2}(\xi)$ is just a bright soliton solution. Applying Eq.(10) to Eq.(9), we construct the general solution of Eq.(9) in the integral form 26$]$

$$
R_{1}=h \int_{P}^{\xi} f \varepsilon(\xi) d \xi-f \int_{Q}^{\xi} h \varepsilon(\xi) d \xi
$$

where $P$ and $Q$ are the integration constants, $f=d R_{0} / d \xi$ and $h=f \int f^{-2} d \xi$ are two linearly independent solutions of Eq.9. for $\varepsilon(\xi)=0$,

$$
\begin{aligned}
f= & \sqrt{\frac{2}{-g_{1}}} D \operatorname{sech}\left[\sqrt{-D}\left(\xi+c_{0}\right)\right] \tanh \left[\sqrt{-D}\left(\xi+c_{0}\right)\right] \\
h= & -\frac{\sqrt{-2 g_{1}}}{8(-D)^{\frac{2}{3}}} \operatorname{sech}\left[\sqrt{-D}\left(\xi+c_{0}\right)\right] \tanh \left[\sqrt{-D}\left(\xi+c_{0}\right)\right] \\
& \times\left\{6 \sqrt{-D}\left(\xi+c_{0}\right)-4 \operatorname{coth}\left[\sqrt{-D}\left(\xi+c_{0}\right)\right]\right. \\
& \left.+\sinh \left[2 \sqrt{-D}\left(\xi+c_{0}\right)\right]\right\} .
\end{aligned}
$$

Generally, the corrected solution (11) is unbounded, because of the unboundedness of $h$ at $\xi=\infty$. However, using the l'Höpital rule, we can easily obtain the sufficient and necessary boundedness condition [26]

$$
I_{ \pm}=\lim _{\xi \rightarrow \pm \infty} \int_{P}^{\xi} f \varepsilon(\xi) d \xi=0 .
$$

It is worth noting that the relation between the Melnikov function $M\left(c_{0}\right)$ and Eq.(13) is $M\left(c_{0}\right)=I_{+}-I_{-}=$ $\int_{-\infty}^{+\infty} f \varepsilon(\xi) d \xi$. Combining $\varepsilon(\xi)$ in Eq. (9) with $R_{0}$ in Eq.(10) and $f$ in Eq.(12) to the integrand, we yield the well-known Melnikov chaos criterion [27]

$$
\begin{aligned}
& M\left(c_{0}\right)=\frac{2 k^{2} \pi}{g_{1}}\left[\tilde{V}_{1} \operatorname{csch}\left(\frac{k \pi}{\sqrt{-D}}\right) \sin \left(2 c_{0} k\right)\right. \\
& \left.+\tilde{V}_{2} \gamma^{2} \operatorname{csch}\left(\frac{\gamma k \pi}{\sqrt{-D}}\right) \sin \left(2 c_{0} \gamma k-2 \phi\right)\right]=0,
\end{aligned}
$$

which indicates the existence of chaos for some $c_{0}$ values. Under the conditions of Eqs.(13) and (14) we can call Eq.(11) the "chaotic solution". Thus inserting Eqs. (10) and (11) into Eq. (7) produces the chaotic bright soliton solution which is the superposition between the soliton and chaotic states and propagates with the velocity of traveling superlattice.

Chaos Probabilities and Regions The Melnikov function is a periodic function of $c_{0}$ for the fixed parameters and rational number $\gamma$, so only the discrete zero points $c_{0}=c_{0 i}$ for $i=1,2, \cdots$ satisfy the chaos criterion $M\left(c_{0 i}\right)=0$. Nevertheless, $c_{0}$ is an integration constant depending on the initial and boundary conditions and cannot be accurately set in experiment. When the stochastic initial and boundary conditions are applied to the numerical or experimental investigations, from Eq. (10) we know that $c_{0}$ takes $c_{0 i}$ values with only a certain probability which is just the probability from soliton to chaos. Defining this probability as the chaos probability $P$, it is clear that the $P$ value should be proportional to the number $n$ of $c_{0 i}$ in one period of $M\left(c_{0}\right)$ and is always smaller than 1. Taking the chaos probability of single lattice case as the referential one $P_{0}$, we have the relation $P=n P_{0} / 2$ between $P$ and $n$, since $n$ is equal to 2 in the single lattice case as in Eq. (14) with $\tilde{V}_{2}=0$.

Now let us see how the chaos probability depends on the parameter regions. As a simple example we firstly 


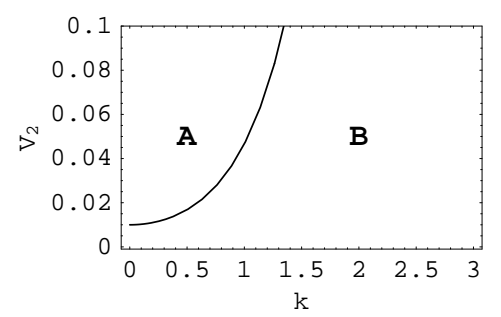

FIG. 1: Plot of the chaotic regions of $\tilde{V}_{2}$ versus $k$ for the dimensionless parameters $\gamma=2, D=-2$ and $\tilde{V}_{1}=0.04$.

consider the case $\phi=0$ and $\gamma=N \geq 2$ with $N$ integer. We rewrite Eq.(14) in the form

$$
M\left(c_{0}\right)=\eta X_{1}\left(c_{0}\right) X_{N}\left(c_{0}\right)=0,
$$

where $\eta=2 k^{2} \pi / g_{1}, \quad X_{1}\left(c_{0}\right)=\sin \left(2 c_{0} k\right)$, and $X_{N}\left(c_{0}\right)=\tilde{V}_{1} \operatorname{csch}(k \pi / \sqrt{-D})+F_{N}\left(c_{0}\right)$ with $F_{N}\left(c_{0}\right)=$ $\tilde{V}_{2} N^{2} \operatorname{csch}\left(\frac{N k \pi}{\sqrt{-D}}\right) \frac{\sin \left(N 2 c_{0} k\right)}{\sin \left(2 c_{0} k\right)}$. Clearly, the $X_{N}\left(c_{0}\right)$ is a periodic function of $c_{0}$ but with different number of zero points in different parameter regions, which can lead to different number $n$ of $c_{0 i}$ and different chaos probabilities. This can be easily evidenced in the case $N=2$. The superlattice of Eq.(1) with $\gamma=2$ has be widely studied [24]. In such a case, the $X_{N}\left(c_{0}\right)$ becomes $X_{2}\left(c_{0}\right)=\tilde{V}_{1} \operatorname{csch}(k \pi / \sqrt{-D})+$ $8 \tilde{V}_{2} \operatorname{csch}(2 k \pi / \sqrt{-D}) \cos \left(2 c_{0} k\right)$ and $M\left(c_{0}\right)$ has period $\pi$. Obviously, two parameter regions with different zero points of $X_{2}\left(c_{0}\right)$ exist and the boundary between them is given by

$$
\tilde{V}_{2}=\tilde{V}_{2 b}=\frac{\tilde{V}_{1} \operatorname{csch}(k \pi / \sqrt{-D})}{8 \operatorname{csch}(2 k \pi / \sqrt{-D})}=\frac{\tilde{V}_{1}}{4} \cosh \frac{k \pi}{\sqrt{-D}} .
$$

As an instance from Eq. (16) with $D=-2, \tilde{V}_{1}=0.04$ the boundary curve on the parameter plane $\left(k, \tilde{V}_{2}\right)$ is plotted as in Fig. 1. The region $A$ above the boundary curve is associated with $\tilde{V}_{2}>\tilde{V}_{2 b}$ and the region $B$ below the boundary curve corresponds to $\tilde{V}_{2}<\tilde{V}_{2 b}$.

In order to show the different numbers $n$ of zero-point $c_{0 i}$ in one period of $M\left(c_{0}\right)$ for the different parameter regions, we make the sketch maps showing the zero points of functions $X_{1}\left(c_{0}\right), X_{2}\left(c_{0}\right)$ and $M\left(c_{0}\right)$ as in Fig. 2. For the single lattice case with $\tilde{V}_{2}=0$ from Fig. 2(a) we see that $X_{2}\left(c_{0}\right)=$ constant, $M\left(c_{0}\right)$ and $X_{1}\left(c_{0}\right)$ have $n=2$ same zero points, indicating the chaos probability $P=$ $n P_{0} / 2=P_{0}$. In Fig. 2(b) with parameters of region $A$, we exhibit that both $X_{1}\left(c_{0}\right)$ and $X_{2}\left(c_{0}\right)$ have two zero points in range $c_{0} \in[0, \pi)$ respectively, and all the zero points are not coincident. This means $n=4$ and the chaos probability $P=n P_{0} / 2=2 P_{0}$ in region $A$. On the boundary between regions $A$ and $B$, from Fig. 2 (c) we can see that two zero points coincide among the three zero points of $X_{1}\left(c_{0}\right)$ and $X_{2}\left(c_{0}\right)$ in one period. Therefore, we have $n=2$ and $P=P_{0}$ on the boundary curve. The same chaos probability appears in region $B$, which is illustrated by Fig. 2(d), where $X_{2}\left(c_{0}\right)$ has no zero point and the number $n=2$ of zero points of $M\left(c_{0}\right)$ agrees with that of $X_{1}\left(c_{0}\right)$.
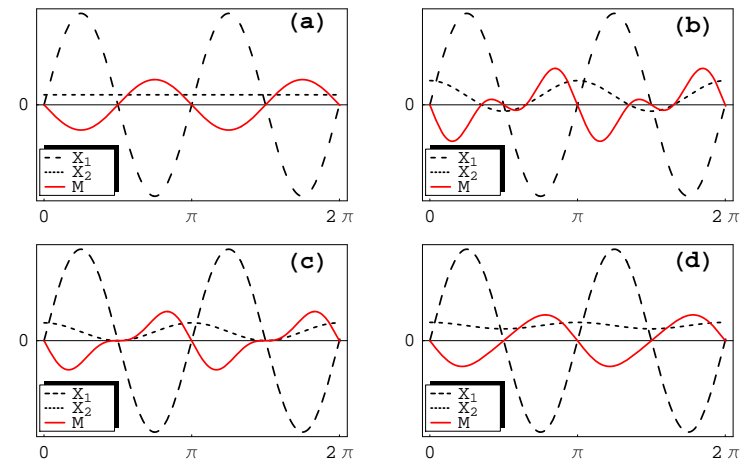

FIG. 2: Sketch maps of functions $X_{1}\left(c_{0}\right)$ (dashed curve), $X_{2}\left(c_{0}\right)$ (doted curve) and $M\left(c_{0}\right)$ (solid curve) versus $c_{0}$ for the parameters $\gamma=2, D=-2, g_{1}=-0.5, k=1, \tilde{V}_{1}=0.04$ and (a) $\tilde{V}_{2}=0$, (b) $\tilde{V}_{2}=0.08>\tilde{V}_{2 b}$, (c) $\tilde{V}_{2}=0.01 \cosh (\pi / \sqrt{2})=0.0466 \approx \tilde{V}_{2 b}$, (d) $\tilde{V}_{2}=0.01<\tilde{V}_{2 b}$, where for each different function the different amplitude scale is adopted for showing the zero points.

The above results can be classified as the two cases:

Case 1. In the double chaotic region $A$ with parameters obeying $\tilde{V}_{2}>\tilde{V}_{2 b}$, the chaos probability reads $P=2 P_{0}$

Case 2. In the chaotic region $B$ and on the boundary curve with parameters obeying $\tilde{V}_{2} \leq \tilde{V}_{2 b}$, the chaos probability equates the referential one, $P=P_{0}$.

To numerically confirm the analytical results, we use the MATHEMATICA code

$$
\begin{aligned}
& T=\pi, e\left[\left\{\text { Rnew }_{\text {,vnew }}\right]:=\{R[T], v[T]\} / .\right. \text { Flatten } \\
& {\left[N D \text { Solve } \left[\left\{R^{\prime}[\xi]==v[\xi], v^{\prime}[\xi]==g_{1} R[\xi]^{3}-D R[\xi]\right.\right.\right.} \\
& +\left(V_{1} \operatorname{Cos}[k \xi]^{2}+V_{2} \operatorname{Cos}[\gamma k \xi]^{2}\right) R[\xi], R[0]==\text { Rnew, } \\
& v[0]==\text { vnew }\},\{R, v\},\{\xi, 0, T\}]] ; \text { Do }\left[\text { pic } c_{i}=\right.\text { ListPlot } \\
& {[\text { Drop }[\text { Nestlist }[e,\{\text { Random }[\text { Real, }\{-2.1,2.1\}],} \\
& \text { Random }[\text { Real, }\{-2.2,2.2\}]\}, 3000], 100],\{i, 1,500\}]
\end{aligned}
$$

to make two groups of Poincaré sections on the equivalent phase space $\left(R, R_{\xi}\right)$ for the parameters used in Fig. 2(b) and Fig. 2(d) and the random initial conditions $\left\{R\left(\xi_{0}\right) \in[-2.1,2.1], R_{\xi}\left(\xi_{0}\right) \in[-2.2,2.2]\right\}$ associated with a suitable range of $c_{0}$. Each of the groups contains five hundreds of Poincaré sections. For the parameters in region $A, 180$ chaotic attractors are found, and the number of chaotic attractors is 85 for the parameters in region $B$. The numerical results show that in the chaotic region $B$ the chaos probability reads $P=P_{0}=85 / 500=0.17$, and in the double chaotic region $A$ the chaos probability becomes $P=180 / 500=0.36 \approx 2 P_{0}$. They well agree with the analytical assertion. The small difference exists between the analytical and numerical results, because of that the strict chaos probability requires more samplings of the Poincaré sections than the used ones.

Then, we take $\phi=0$ and $\gamma=N=3,4,5$ into account and find that for any $N$ the parameter space can be divided into several parts with different chaos probabilities. 
The lowest chaos probability is $P_{0}$ and the highest one arrives at $N P_{0}$. Moreover, the number of chaotic regions increases with $N$.

Control of Chaos Probabilities Controlling the chaos probability to zero is important for some cases one requires suppressing chaos. On the other hand, for the secure chaos-based cryptosystems 22, 23] one calls for higher chaotic probability. Given the above-mentioned results, we can decrease or raise the chaos probability by adjusting the parameters into different chaotic regions. In order to suppress chaos, at first we let the parameters enter the region of the lowest chaos probability (e.g. the above region $B)$, where only $X_{1}\left(c_{0}\right)=\sin \left(2 c_{0} k\right)$ has zero points. For such a parameter region if a chaotic state is observed experimentally, which implies $2 c_{0} k=i \pi$ for $i=1,2, \cdots$ and the first term of Eq. (14) vanishing. Then we can adopt the two different methods to eliminate chaos:

Method 1. We take $\phi=0$ and vary $\gamma$ from an integer to an irrational number suddenly such that the second term in Eq. (14), $\sin 2 \gamma c_{0} k=\sin i \gamma \pi$, does not vanish and the Melnikov chaos criterion $M\left(c_{0}\right)=0$ cannot be fulfilled, namely $n=0$ and $P=n P_{0} / 2=0$.

Method 2. Let $\gamma$ be equal to 2 and suddenly change $\phi$ from zero to nonzero, say $\pi / 4$, the nonzero Melnikov function can also be obtained, that leads the chaos probability to zero.

The operation of method 1 can be performed by switch- ing off the second lattice with $\gamma=$ integer suddenly and switching on the third lattice with $\gamma=$ irrational number simultaneously. Similarly, we can complete the operation of method 2 by a new lattice of phase difference $\pi / 4$ with the second lattice. The analysis offers an effective technique for suppressing spatiotemporal chaos experimentally.

In conclusion, we have investigated a BEC system loaded into a weak moving optical superlattice created by interference between two lattices of different depths and wave vectors. The superlattice separates the chaotic region into several parts with different chaos probabilities, which are studied analytically and numerically. Moreover, for a fixed first lattice, the modulation of the secondary lattice can transform the chaos probability to zero or higher one. Our results suggest a feasible method for suppressing or strengthening chaos experimentally. When the zero traveling velocity $v_{L}=0$ is set, the results fit the corresponding static BEC system. The used method could also be easily extended to the controls of other chaotic systems.

\section{Acknowledgments}

This work was supported by the National Natural Science Foundation of China under Grant No. 10575034.
[1] F. Kh. Abdullaev, and R. A. Kraenkel, Phys. Rev. A 62, 023613 (2000).

[2] C. Zhang, J. Liu, M. G. Raizen, and Q. Niu, Phys. Rev. Lett. 93, 074101 (2004).

[3] F Kh Abdullaev, and R Galimzyanov, J. Phys. B 36, 1099 (2003).

[4] T. Mayteevarunyoo, B. A. Malomed, and M. Krairiksh, Phys. Rev. A 76, 053612 (2007).

[5] K. Nozaki, and N. Bekki, Phys. Rev. Lett. 50, 1226 (1983).

[6] H. T. Moon, and M. V. Goldman, Phys. Rev. Lett. 53, 1821 (1984).

[7] R. Scharf and A. R. Bishop, Phys. Rev. A 46, R2973 (1992).

[8] P. V. Elyutin, A. V. Buryak, V. V. Gubernov, R. A. Sammut, and I. N. Towers, Phys. Rev. E 64, 016607 (2001).

[9] F. Kh. Abdullaev, E. N. Tsoy, B. A. Malomed, and R. A. Kraenkel, Phys. Rev. A 68, 053606 (2003).

[10] A. D. Martin, C. S. Adams, and S. A. Gardiner, Phys. Rev. Lett. 98, 020402 (2007).

[11] R. Roth and K. Burnett, J. Opt. B 5, S50 (2003).

[12] C. Huang and W. Wu, Phys. Rev. A 72, 065601 (2005).

[13] L. Sanchez-Palencia and L. Santos, Phys. Rev. A 72, 053607 (2005).

[14] A. A. Sukhorukov, Phys. Rev. Lett. 96, 113902 (2006).

[15] J. E. Lye, L. Fallani, C. Fort, V. Guarrera, M. Modugno, D. S. Wiersma, and M. Inguscio, Phys. Rev. A 75, 061603(R) (2007).
[16] D. Shechtman, I. Blech, D. Gratias and J. W. Cahn, Phys. Rev. Lett. 53, 1951 (1984).

[17] J. Hecker Denschlag, J. E. Simsarian, H. Häfner, C. McKenzie, A. Browaeys, D. Cho, K. Helmerson, S. L. Rolston, and W. D. Phillips, J. Phys. B 35, 3095 (2002).

[18] L. Fallani1, F. S. Cataliotti1, J. Catani, C. Fort, M. Modugno, M. Zawada, and M. Inguscio, Phys. Rev. Lett. 91, 240405 (2003).

[19] B. Eiermann, Th. Anker, M. Albiez, M. Taglieber, P. Treutlein, K. P. Marzlin, and M. K. Oberthaler, Phys. Rev. Lett. 92, 230401 (2004).

[20] G. Chong, W. Hai, and Q. Xie, Phys. Rev. E 70, 036213 (2004).

[21] F. Li, W. Shu, J. Jiang, H. Luo, and Z. Ren, Eur. Phys. J. D 41, 355(2007).

[22] K. M. Cuomo and A. V. Oppenheim, Phys. Rev. Lett. 71, 65 (1993).

[23] X. Li, H. Zhang, Y. Xue, and G. Hu, Phys. Rev. E71, 016216 (2005).

[24] P. J. Y. Louis, E. A. Ostrovskaya, and Y. S. Kivshar, Phys. Rev. A 71, 023612 (2005).

[25] S. A. Gardiner, D. Jaksch, R. Dum, J. I. Cirac, and P. Zoller, Phys. Rev. A 62, 023612 (2000).

[26] W. Hai, C. Lee, G. Chong and L. Shi, Phys. Rev. E 66, 026202(2002); C. Lee, W. Hai, L. Shi, X. Zhu and K. Gao, Phys. Rev. A64, 053604(2001); W. Hai, X. Liu, J. Fang, X. Zhang, W. Huang, G. Chong, Phys. Lett. A 275, 54 (2000).

[27] V. K. Melnikov, Trans. Moscow Math. Soc. 12, 1 (1963). 